\title{
Patogenicidade de Beauveria bassiana sobre estágios imaturos de Stomoxys calcitrans
}

\author{
Pathogenicity of Beauveria bassiana on immature stages of Stomoxys calcitrans
}

\author{
Ana Paula Rodrigues Moraes ${ }^{\mathrm{I}}$ Vânia Rita Elias Pinheiro Bittencourt ${ }^{\mathrm{II}}$ \\ Avelino José Bittencourt ${ }^{\mathrm{III}}$
}

\section{RESUMO}

Por ser um díptero hematófago, a mosca Stomoxys calcitrans (Linnaeus, 1758) ocasiona grandes perdas na pecuária brasileira. Atualmente, pesquisas têm sido direcionadas para métodos de controle que reduzam a utilização de inseticidas químicos e que preservem o meio ambiente. Este estudo avaliou o potencial entomopatogênico dos isolados CG 138 e CG 228 de Beauveria bassiana sobre ovos, larvas e pupas de $\boldsymbol{S}$. calcitrans em condições ambientais e o isolado ESALQ 986 de B. bassiana em ovos, nas mesmas condições. Para cada isolado fúngico, foram utilizadas as concentrações de $2 \times 10^{6}, 2 \times 10^{7}$ e $2 \times 10^{8}$ conídios $\mathrm{ml}^{-1}$. Os resultados sugerem que os isolados CG 138, CG 228 e ESALQ 986 apresentaram efeito saprofítico, pois não afetaram significativamente o desenvolvimento dos estágios imaturos da mosca dos estábulos, comprometendo dessa forma sua utilização como agente entomopatogênico.

Palavras-chave: mosca dos estábulos, fungo entomopatogênico, controle biológico.

\section{ABSTRACT}

Because it is a hemathofagic diptera, the Stomoxys calcitrans (Linnaeus, 1758) inflicts great losses in the Brazilian cattle. Nowadays, researches have been directed to the control methods that reduce the use of chemical insecticides and preserve the environment. This study evaluated the entomopathogenic potential of the isolated CG 138 and CG 228 of Beauveria bassiana on eggs, larvae and pupae of $\boldsymbol{S}$. calcitrans in environmental conditions and the isolated ESALQ 986 of B. bassiana in eggs. For each fungal isolated were used concentrations of $2 \times 10^{6}, 2 \times 10^{7}$ and $2 \times 10^{8}$ conidia $\mathrm{ml}^{-1}$. The results suggest that the isolated CG 138, CG 228, ESALQ 986 present a saprophytic effect, because they did not affect significantly the development of immature stages of the stable flies, affecting this way its use as entomopathogenic agent.

Key words: Stable fly, entomopathogenic fungi, biologic control.

\section{INTRODUÇÃO}

O hematofagismo realizado pela mosca Stomoxys calcitrans (Linnaeus, 1758) ocasiona grande estresse aos animais parasitados (GUIMARÃES, 1983; MIHOK \& CLAUSEN, 1996; BITTENCOURT \& MOYA-BORJA, 2000), possibilita a transmissão de diferentes agentes patogênicos (TARRY \& CARROLL, 1988) e o desenvolvimento de dermatites ulcerativas (WHITE \& BOURDEAU, 1995). Segundo CAMPBELL et al. (1987), a presença de 35 moscas no membro de um bovino é suficiente para ocasionar redução de $12 \%$ na conversão alimentar e $20 \%$ em queda de peso.

SOULSBY (1987) destaca que os maiores problemas ocasionados pela mosca dos estábulos estão relacionados aos locais onde podem ser encontradas condições adequadas para seu desenvolvimento. Com isso, as medidas de controle devem ser direcionadas aos pontos onde exista acúmulo de dejetos vegetais em decomposição associados à urina e a fezes de animais, com intuito de racionalizar e reduzir o uso de inseticidas químicos.

'Programa de Pós-graduação em Ciências Veterinárias, Instituto de Veterinária, UFRRJ, Seropédica, RJ, Brasil.

IIDepartamento de Parasitologia Veterinária, Instituto de Veterinária, UFRRJ, Seropédica, RJ, Brasil.

IIIDepartamento de Medicina e Cirurgia Veterinária, Instituto de Veterinária, UFRRJ, BR 465, Km 7, 23890-000, Seropédica, RJ, Brasil. E-mail: bittenc@ufrrj.br. Autor para correspondência. 
Essas medidas devem ocasionar o menor impacto ambiental possível e controlar os insetos a níveis populacionais que não afetem economicamente a produção (ALVES, 1998).

Segundo GUIMARÃES (1983), S. calcitrans apresenta maior tolerância aos inseticidas químicos quando comparada a outras moscas, pois, como possui alimentação interrompida, permanece por curtos períodos nos animais. Por isso, a aplicação de inseticidas biológicos ou associados a inseticidas químicos, nos locais onde seus estágios imaturos se desenvolvem, pode apresentar melhores resultados.

O fungo entomopatogênico Beauveria bassiana (Balsamo) Vuillemin, 1912 pode ser encontrado em amostras de solo e em grande variedade de insetos e é uma das espécies mais estudadas no controle biológico de artrópodes (ALVES, 1998). Tanto B. bassiana, como Metarhizium anisopliae são eficientes para o controle de moscas, como Glossina spp., quando incorporados ao meio de desenvolvimento dos estágios imaturos (KAAYA \& MUNYINYI, 1995).

Tratamentos experimentais com diferentes microrganismos têm como objetivo selecionar agentes entomopatogênicos mais adequados para o controle biológico de determinada praga, em que devem ser considerados os seguintes fatores: a segurança de utilização, a especificidade, a patogenicidade, a capacidade de produção industrial e a viabilidade após exposição a condições ambientais (ALVES, 1998). FERNANDES (2007) destaca que a maior parte dos estudos, em que se avalia o efeito entomopatogênico sobre o hospedeiro-alvo, é realizada em condições ideais de laboratório, o que poderia superestimar sua ação em condições naturais. Dessa forma, foi avaliada neste estudo a ação dos isolados CG 138, CG 228 e ESALQ 986 de $\boldsymbol{B}$. bassiana sobre estágios imaturos de S. calcitrans em condições de temperatura e umidade naturais.

\section{MATERIAL E MÉTODOS}

As moscas adultas foram mantidas em gaiolas plásticas, onde se forneceu sangue bovino com citrato de sódio (0,38\%). Para manutenção dos ovos, larvas e pupas, foi utilizada dieta adaptada por MORAES (2007). Estes foram mantidos em câmara climatizada do tipo B.O.D., a $26 \pm 1^{\circ} \mathrm{C}$ e $70-80 \%$ de umidade relativa (U.R.).

A dieta de criação utilizada nos bioensaios foi composta por cana-de-açúcar (66g), farelo de trigo (25g), carne moída bovina (8g), bicarbonato de sódio (1g) e água destilada (127ml), acondicionados em becker de $600 \mathrm{ml}$ e esterilizados em autoclave por 20 minutos, a $120^{\circ} \mathrm{C}$ (MORAES, 2007).

A partir de estudo realizado por FERNANDES (2007), os fungos foram selecionados segundo a viabilidade conidial após exposição a diferentes temperaturas $\left(5^{\circ} \mathrm{C} \mathrm{e} 45^{\circ} \mathrm{C}\right)$ e à radiação UV-B, visto que são fatores ambientais limitantes na utilização desses agentes no controle biológico de artrópodes (SMITS et al., 2003). Os isolados CG 138 (Recife - PE) e CG 228 (Brasília, DF) foram obtidos de coleópteras da família Curculionidae e Chrysomelidae, respectivamente, enquanto o isolado ESALQ 986 (Piracicaba, SP) foi isolado de carrapato da família Ixodidae. Os três isolados apresentaram elevada tolerância à radiação UV-B e às temperaturas de $45^{\circ} \mathrm{C}$ (uma e duas horas) e $5^{\circ} \mathrm{C}$ (15 dias), com exceção do isolado ESALQ 986, que foi menos tolerante à temperatura elevada.

Os isolados CG 138, CG 228 e ESALQ 986 de

B. bassiana foram semeados em placas de Petri contendo ágar batata dextrose (BDA) e extrato de levedura (EL) - 1\%(ALVES, 1998). Estes foram mantidos em câmara climatizada tipo B.O.D. (25ㄷ, $70-80 \%$ U.R.), por 15 dias, em ausência de luz. Por apresentar elevada virulência contra artrópodes (FERNANDES, 2007), o isolado ESALQ 986 de B. bassiana foi utilizado para comprovar os resultados verificados com os outros dois fungos.

Para o preparo das suspensões nas concentrações de $2 \times 10^{8}, 10^{7}$ e $10^{6}$ conídios $\mathrm{ml}^{-1}$, os fungos foram transferidos das placas de Petri com auxílio de lâmina de bisturi para a solução estéril de água destilada e Tween 80 (0,01\%) (agitação por um minuto), realizando-se a contagem dos conídios em câmara de Neubauer e microscópio óptico. A avaliação da viabilidade conidial foi confirmada pela inoculação das suspensões em placa de Petri contendo BDA e EL. Após 24 horas, realizou-se contagem dos conídios germinados (ALVES, 1998).

Os isolados CG 138 e CG 228 foram utilizados em ovos, larvas e pupas, enquanto que o isolado ESALQ 986 foi utilizado apenas nos ovos para confirmação do efeito observado com os outros dois isolados. Grupos de 80 ovos, 30 larvas e 30 pupas foram depositados separadamente sobre a dieta, e $10 \mathrm{ml}$ de cada suspensão fúngica foi aspergida com auxílio de um borrifador plástico, enquanto que os grupos de controle foram expostos apenas ao diluente. Em seguida, os recipientes foram cobertos com tecido de algodão (CHRISTMAS, 1970) e abrigados em local protegido da irradiação solar direta e da chuva, permitindo a exposição às condições naturais de temperatura e umidade. Após 30 dias, realizaram-se a contagem de moscas emergidas e a identificação dos fungos utilizados nos bioensaios (ALVES, 1998). 
Para aferição diária da temperatura e umidade ambiental, foi utilizado termohigrômetro digital. Os experimentos foram realizados duas vezes e em dias diferentes para confiabilidade dos dados. Os resultados foram analisados pelo teste de Qui-quadrado $(\mathrm{P} \leq 0,05)$ (SAMPAIO, 2002), e a mortalidade dos grupos de controle foi corrigida pela Fórmula de Abbott (ALVES, 1998).

\section{RESULTADOS E DISCUSSÃO}

No presente estudo, foi verificado baixo percentual de mortalidade dos ovos expostos à concentração $2 \times 10^{8}$ conídios $\mathrm{ml}^{-1}$ nas duas repetições realizadas (Tabela 1). Com o isolado CG 138 de $\boldsymbol{B}$. bassiana, foram obtidos apenas 20,0 e 22,5\% de mortalidade e, com isolado CG 228, foram obtidos 22,5 e 21,2\%. Baixos percentuais também foram verificados com isolado ESALQ 986, mesmo sendo considerado um fungo de elevada patogenicidade (FERNANDES, 2007). Esses resultados diferiram dos descritos por MORAES et al. (2008), que obtiveram 100,0\% de inviabilidade dos ovos de $\boldsymbol{S}$. calcitrans expostos ao fungo Metarhizium anisopliae pelo método de imersão ou aspersão com mesma concentração fúngica. Não foi obtida diferença significativa entre os grupos expostos ao fungo $\boldsymbol{B}$. bassiana e o grupo controle.

Os isolados CG 138 e CG 228 de $\boldsymbol{B}$. bassiana não foram capazes de afetar significativamente os percentuais de mortalidade nos experimentos com larvas de $\boldsymbol{S}$. calcitrans em todas as concentrações fúngicas utilizadas, pois os grupos tratados não diferiram dos 36,6 e 33,3\% de mortalidade de larvas dos grupos de controle, sendo observados os mesmos percentuais quando as larvas foram expostas à concentração $2 \times 10^{8}$ conídios $\mathrm{ml}^{-1}$. Percentuais de mortalidade pupal próximos aos verificados nos grupos de controle também foram observados no estudo (Tabela 1), demonstrando a ineficácia desses isolados fúngicos em controlar a emergência de $\boldsymbol{S}$. calcitrans. Resultados semelhantes foram obtidos por outros autores, a exemplo da baixa efetividade do fungo $\boldsymbol{M}$. anisopliae em impedir o desenvolvimento de larvas e pupas de $\boldsymbol{S}$. calcitrans (MORAES et al., 2008), em que os estágios imaturos foram imersos em concentrações fúngicas similares às condições utilizadas no presente estudo. BERNAL et al. (2003) verificaram que suspensões de $\boldsymbol{M}$. anisopliae nas concentrações de $1 \times 10^{2}, 1 \times 10^{4}, 1 \times 10^{6}$ e $1 \times 10^{8}$ também não foram capazes de afetar a viabilidade de larvas de segundo instar de S. calcitrans. ANGEL-SAHAGÚN et al. (2005) verificaram susceptibilidade variável de ovos, larvas e pupas de Haematobia irritans quando expostos a diferentes isolados fúngicos de $\boldsymbol{B}$. bassiana. Os autores destacaram que o efeito patogênico dos fungos estaria diretamente relacionado ao isolado empregado.

Os resultados obtidos nas duas repetições do presente estudo demonstraram que os isolados CG

Tabela 1 - Percentual de mortalidade de ovos, larvas e pupas de Stomoxys calcitrans expostos aos isolados CG 138, CG 228 e ESALQ 986 de Beauveria bassiana em condições naturais de temperatura e umidade.

\begin{tabular}{|c|c|c|c|c|c|c|c|c|c|c|c|c|}
\hline \multirow[b]{3}{*}{$\begin{array}{l}\text { Tratamento } \\
\left(\text { conídios ml }{ }^{-1} \text { ) }\right.\end{array}$} & \multicolumn{6}{|c|}{ Primeiro bioensaio } & \multicolumn{6}{|c|}{ Segundo bioensaio } \\
\hline & \multicolumn{2}{|c|}{ Ovo* } & \multicolumn{2}{|c|}{ Larva* } & \multicolumn{2}{|c|}{ Pupa* } & \multicolumn{2}{|c|}{ Ovo* } & \multicolumn{2}{|c|}{ Larva* } & \multicolumn{2}{|c|}{ Pupa* } \\
\hline & M (\%) & $\begin{array}{l}\text { MC } \\
(\%)\end{array}$ & M (\%) & $\begin{array}{l}\text { MC } \\
(\%)\end{array}$ & M (\%) & $\begin{array}{l}\text { MC } \\
(\%)\end{array}$ & M (\%) & $\begin{array}{l}\text { MC } \\
(\%)\end{array}$ & M (\%) & $\begin{array}{l}\text { MC } \\
(\%)\end{array}$ & M (\%) & $\begin{array}{l}\text { MC } \\
(\%)\end{array}$ \\
\hline Controle & 37,5 & 0 & 36,7 & 0 & 20,0 & 0 & 23,7 & 0 & 33,3 & 0 & 13,3 & 0 \\
\hline & --- & - & -----CG & 38------ & -------- & ---- & $---\cdot$ & - & ----CG & 38---- & ------ & ----- \\
\hline $2 \times 10^{6}$ & 47,5 & 16,0 & 16,7 & $-31,6$ & 16,7 & $-4,2$ & 37,5 & 18,0 & 36,7 & 5,0 & 23,3 & 11,5 \\
\hline $2 \times 10^{7}$ & 47,5 & 16,0 & 33,3 & $-5,2$ & 16,7 & $-4,2$ & 36,2 & 16,4 & 36,7 & 5,0 & 20,0 & 7,7 \\
\hline \multirow[t]{2}{*}{$2 \times 10^{8}$} & 20,0 & $-28,0$ & 36,7 & 0 & 16,7 & $-4,2$ & 22,5 & $-1,6$ & 33,3 & 0 & 20,0 & 7,7 \\
\hline & \multicolumn{6}{|c|}{-----CG 228----. } & \multicolumn{4}{|c|}{---------------------------CG 228-- } & & ----- \\
\hline $2 \times 10^{6}$ & 25,0 & $-20,0$ & 46,7 & 15,8 & 20,0 & 0 & 20,0 & $-4,9$ & 26,7 & $-10,0$ & 23,3 & 11,5 \\
\hline $2 \times 10^{7}$ & 25,0 & $-20,0$ & 46,7 & 15,8 & 20,0 & 0 & 22,5 & $-1,6$ & 26,7 & $-10,0$ & 26,7 & 15,4 \\
\hline \multirow[t]{2}{*}{$2 \times 10^{8}$} & 22,5 & $-24,0$ & 33,3 & $-5,2$ & 23,3 & 4,2 & 21,2 & $-3,3$ & 23,3 & $-15,0$ & 16,7 & 3,8 \\
\hline & \multicolumn{6}{|c|}{--ESALQ 986--------------------------- } & -------- & & ---ESAI & 986-- & --- & ----- \\
\hline Controle & 25,0 & 0 & - & - & - & - & 28,7 & 0 & - & - & - & - \\
\hline $2 \times 10^{6}$ & 21,2 & $-5,0$ & - & - & - & - & 28,7 & 0 & - & - & - & - \\
\hline $2 \times 10^{7}$ & 20,0 & $-6,7$ & - & - & - & - & 38,7 & 13,9 & - & - & - & - \\
\hline $2 \times 10^{8}$ & 13,7 & $-15,0$ & - & - & - & - & 28,7 & 0 & - & - & - & - \\
\hline
\end{tabular}

M=mortalidade; MC=Mortalidade corrigida pela fórmula de Abbott; - = Não avaliado. * = Não houve diferença significativa entre os grupos expostos aos isolados fúngicos e o grupo de controle. 
138 e CG 228 de $\boldsymbol{B}$. bassiana não apresentaram o potencial entomopatogênico esperado (Tabela 1), visto que a mortalidade dos grupos tratados foi menor ou igual à mortalidade obtida nos grupos de controle, independente do estágio imaturo avaliado. Em poucas situações, foram observados percentuais de mortalidade maiores que aqueles dos grupos de controle, porém a diferença não foi significativa. A utilização do isolado ESALQ 986 teve por finalidade confirmar se a reduzida mortalidade observada em ovos seria reproduzida, o que foi confirmado pelos resultados obtidos nos dois bioensaios realizados com esse isolado (Tabela 1).

Em estudo de controle biológico de moscas, WATSON et al., (1995) destacaram a tolerância de $\boldsymbol{S}$. calcitrans ao fungo $\boldsymbol{B}$. bassiana, pois foram obtidos menores percentuais de mortalidade quando comparada à mosca doméstica. Esses mesmos autores relataram que o ambiente extremamente contaminado, onde os estágios imaturos de $\boldsymbol{S}$. calcitrans se desenvolvem, estimularia seus mecanismos de defesa e que a composição de sua cutícula resultaria em distinta susceptibilidade à infecção fúngica. Outros autores destacaram que a fermentação da matéria orgânica realizada por diferentes microrganismos favoreceria o desenvolvimento dos estágios imaturos de $\boldsymbol{S}$. calcitrans (SCHMIDTMANN \& MARTIN, 1992; PEROTTI et al., 2001; ROMERO et al., 2006).

Logo, para que um fungo possa atuar sobre os estágios imaturos de moscas, este deve possuir mecanismos que promovam elevada patogenicidade ou que anulem os fatores antimicrobianos do inseto. Pode-se verificar, no presente estudo, que os isolados selecionados capazes de tolerar as adversidades ambientais não foram eficientes para inviabilizar o desenvolvimento dos estágios imaturos da mosca dos estábulos, mesmo quando utilizado um isolado com elevada patogenicidade para artrópodes, como o ESALQ986(FERNANDES, 2007).

Um fator que poderia influenciar a capacidade entomopatogênica dos isolados seria o potencial de germinação dos conídios após o preparo das suspensões e a permanência desses isolados no ambiente. Na avaliação de viabilidade conidial das suspensões, foi possível verificar 100,0\% de conídios viáveis nos três isolados utilizados. Também foi observado intenso desenvolvimento fúngico nos recipientes contendo meio de desenvolvimento larval, onde os experimentos foram realizados. Os valores médios de temperatura e umidade (máxima e mínima) verificados durante o período dos bioensaios $\left(29,53^{\circ} \mathrm{C}\right.$ - 89,73\% U.R. e $19,66^{\circ} \mathrm{C}$ - 52,33\% U.R.) não são considerados como capazes de inviabilizar a ação dos fungos (FERNANDES, 2007). Os resultados observados indicam que a ineficácia dos fungos utilizados no presente estudo não estaria relacionada com a incapacidade de germinação de seus conídios.

Outro fator relacionado à ineficácia dos isolados de $\boldsymbol{B}$. bassiana para o controle dos estágios imaturos de $\boldsymbol{S}$. calcitrans seria a ausência de nutrientes necessários para a germinação dos conídios sobre a cutícula. HUNT et al. (1984) mencionam que os nutrientes disponibilizados nos meios de cultura para o desenvolvimento conidial nem sempre são encontrados nas cutículas dos hospedeiros e que poucos estudos sobre sua composição são relatados. Segundo St. LEGER et al. (1998), o pH do meio seria um dos principais fatores que influenciariam a produção das diferentes enzimas fúngicas, que podem atuar no processo de degradação da cutícula do hospedeiro, sendo necessários mais estudos que relacionem a variação de $\mathrm{pH}$ do meio, onde são encontrados os estágios imaturos de $\boldsymbol{S}$. calcitrans e a ativação das enzimas fúngicas responsáveis pelo processo de penetração da cutícula.

Diferentes estudos vêm sendo desenvolvidos para verificar os fatores que determinam a ineficácia entomopatogênica de certos fungos, como a inibição de proteases e quitinases (VILCINSKAS \& WEDDE, 1997) e a presença de determinados ácidos graxos (SMITH \& GRULA, 1982; HUNT, 1986; BARNES \& MOORE, 1997; JAMES et al., 2003), mas a relação desses fatores com a mosca $\boldsymbol{S}$. calcitrans ainda não são descritos.

\section{CONCLUSÃO}

Os resultados demonstraram que os isolados utilizados no presente estudo não foram eficazes para controlar significativamente os estágios imaturos de $\boldsymbol{S}$. calcitrans. Há a necessidade de se testar quais microrganismos podem ser efetivos no controle biológico de $\boldsymbol{S}$. calcitrans, visto que existem fatores relacionados ao fungo e a seus estágios imaturos que contribuem para a tolerância aos diferentes isolados de $\boldsymbol{B}$. bassiana. Além disso, novos estudos devem ser desenvolvidos para constatar quais fatores contribuem para que ovos, larvas e pupas de $\boldsymbol{S}$. calcitrans possam se desenvolver em ambientes com grande diversidade microbiana.

\section{REFERÊNCIAS}

ANGEL-SAHAGÚN, C.A. et al. Susceptibility of biological stages of the horn fly, Haematobia irritans, to entomopathogenic fungi (Hyphomycetes). Journal of Insect Science, v.50, p.1-8, 2005. Disponível em: <http://www.insectscience.org/5.50/i1536- 
2442-2005-50>.pdf. Acesso em: 04 jul. 2010. doi: 10.1673/ 1536-2442(2005)5[1:SOBSOT]2.0.CO;2.

ALVES, S.B. Controle microbiano de insetos. São Paulo: FEALQ, 1998. 1163p.

BARNES, S.E.; MOORE, D. The effect of fatty, organic or phenolic acids on the germination of conidia of Metarhizium flavoviride. Mycological Research, v.101, n.6, p.662-666, 1997. Disponível em: <http://www.sciencedirect.com/ science?_ob=ArticleURL\&_udi=B7XMR-4RS5040-

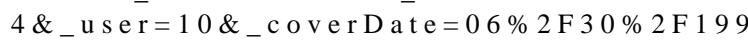
$7 \&$ \& rdoc $=4 \&$ \& $\mathrm{mt}=$ high\&_orig=browse\&_srch $=$ doc info (\%23toc\%2329677\%231997\%23998989993 \%23679805\%23FLP\%23display\%23Volume)\&_cdi=2967 7\&_sort=d\&_docanchor=\&_ct $=168 \_$_acct $=\mathrm{C} 000050221$ \&_version $=1$ \&_url Version=0\&_userid=10\&md5=a2518640106d7b46ef179cee0f5745fb $>$. Acesso em: 04 jul. 2010. doi:10.1017/S0953756296003152.

BERNAL, E.J. et al. Control biologico de la mosca del estábulo Stomoxys calcitrans com el hongo entomopatogeno Metarhizium anisopliae. Revista Colombiana de Ciencias Pecuaria, v.16, supl, p.54, 2003. Disponível em: <http://kogi.udea.edu.co/revista/ 16/16-s-12.pdf\#search=Stomoxys\%20calcitrans\%20Metarhizium>. Acesso em: 17 mar. 2005.

BITTENCOURT, A.J.; MOYA-BORJA, G.E. Flutuação sazonal de Stomoxys calcitrans em bovinos e eqüinos no Município de Espírito Santo do Pinhal. Revista Universidade Rural Série Ciências da Vida, v.22, supl, p.101-106, 2000.

CAMPBELL, J.B. et al. Effects of stable flies (Diptera: Muscidae) on weight gain and feed efficiency of feedlot cattle. Journal of Economic Entomology, v.80, n.1, p.117-119, 1987.

CHRISTMAS, P.E. Laboratory rearing of the biting fly Stomoxys calcitrans (Diptera: Muscidae). New Zealand Entomologist, v.4, p.45-49, 1970. Disponível em: <http:// www.ento.org.nz/nzentomologist/free_issues/volume\%204-445-49.pdf>. Acesso em: 13 mar. 2005.

FERNANDES, E.K.K. Caracterização e seleção de isolados de Beauveria bassiana para o controle microbiano do carrapato Boophilus microplus. 2007. 123f. Tese (Doutorado em Ciências Veterinárias) - Universidade Federal Rural do Rio de Janeiro, Seropédica, RJ.

GUIMARÃES, J.H. Moscas - Biologia, ecologia e controle. Agroquímica Ciba-Geigy, n.21, p.20-26, 1983.

HUNT, D.W.A. Absence of fatty acid germination inhibitors for conidia of Beauveria bassiana on the integument of the bark beetle Dendroctonus ponderosae (Coleoptera: Scolytidae). Canadian Entomologist, v.118, n.8, p.837-838, 1986. Disponível em: <http://pubservices.nrc-cnrc.ca/rp-ps/ inDetail.jsp?jcode $=$ ent $\&$ lang $=$ eng\&vol $=118 \& i s=8>$. Acesso em: 04 jul. 2010.

HUNT, D.W.A. et al. Nutrient-mediated germination of Beauveria bassiana conidia on the integument of the bark beetle Dendroctonus ponderosae (Coleoptera: Scolytidae). Journal of Invertebrate Pathology, v.44, n.3, p.304-314, 1984. Disponível em: <http://www.sciencedirect.com/ science?_ob=PublicationURL\&_tockey=\%23TOC $\% 236888 \% 23198$ $4 \% 23999559996 \% 23542247 \% 23$ F L P \% 23 \&_c
di=6888\&_pubType $=$ J\&_auth $=$ y\&_acct $=$ C000050221\&_ version $=1 \&$ _url Version $=0 \&$ \&_userid $=10 \&$ m d 5 =3f5599a1cb4e70262fb7e2e65bea054a>. Acesso em: 04 jul. 2010. doi: 10.1016/0022-2011(84)90028-4.

JAMES, R.R. et al. Cuticular lipds and silverleaf whitefly stage affect conidial germination of Beauveria bassiana and Paecilomyces fumosoroseus. Journal of Invertebrate Pathology, v.84, p.67-74, 2003. Disponível em: <http:// ddr.nal.usda.gov/bitstream/10113/17031/1/IND43748995.pdf > . Acesso em: 04 jul.2010. doi:10.1016/j.jip.2003.08.006.

KAAYA, G.P.; MUNYINYI, D.M. Biocontrol potential of the entomogenous fungi Beauveria bassiana and Metarhizium anisopliae for tsetse flies (Glossina spp.) at developmental sites. Journal of Invertebrate Pathology, v.66, p.237-241, 1995. Disponível em: <http://www.sciencedirect.com/ science?_ob=ArticleURL\&_udi=B6WJV-45S94DS$5 \&$ \&user $=10 \&$ \&_coverDate $=11 \% 2$ F $30 \% 2$ F $1995 \&$ \&doc $=$ 18 _fmt=high\&_orig=search\&_sort $=\mathrm{d} \&$ \&docanchor $=\mathbf{\&}$ view $=$ c\&_searchStrId=1391109999\&_rerunOrigin=google \&_acct $=$ C 000050221 \&_version $=1$ \&_urlversion $=0$ \&_userid $=10 \& m d 5=0$ ae47dcd2aadc027562c32d41a0706d8 $>$. Acesso em: 04 jul. 2010. doi:10.1006/jipa.1995.1095.

MIHOK, S.; CLAUSEN, P.H. Feeding habits of Stomoxys spp. stable flies in a Kenyan forest. Medical and Veterinary Entomology, v.10, n.4, p.392-394, 1996. Disponível em: <http://www3.interscience.wiley.com/journal/120146656/ issue $>$. Acesso em: 04 jul. 2010. doi: 10.1111/j.13652915.1996.tb00762.x.

MORAES, A.P.R. Stomoxys calcitrans: estabelecimento de colônia e efeito de Metarhizium anisopliae sobre seus estágios imaturos. 2007. 52f. Dissertação (Mestrado em Ciências Veterinárias) - Universidade Federal Rural do Rio de Janeiro, Seropédica, RJ.

MORAES, A.P.R. et al. Virulence of Metarhizium anisopliae to eggs and immature stages of Stomoxys calcitrans. Animal Biodiversity and Emerging Diseases: Annals New York Academy of Sciences, v.1149, p.384-387, 2008. Disponível em: <http://www3.interscience.wiley.com/journal/121569862/ issue $>$. Acesso em: 04 jul. 2010. doi: 10.1196/annals.1428.008.

PEROTTI, T.M.A. et al. Growth and survival of immature Haematobia irritans (Diptera: Muscidae) is influenced by bacteria isolated from cattle manure and conspecific larvae. Journal of Medical Entomology, v.38, n.2, p.180-187, 2001. Disponível em: <http://www.bioone.org/toc/ment/38/2>. Acesso em: 04 jul. 2010. doi: 10.1603/0022-2585-38.2.180.

ROMERO, A. et al. Role of bacteria in the oviposition behaviour and larval development of stable flies. Medical and Veterinary Entomology, v.20, p.115-121, 2006. Disponível em: <http://www3.interscience.wiley.com/journal/118628202/ issue $>$. Acesso em: 04 jul. 2010. doi: 10.1111/j.13652915.2006.00602.x.

SAMPAIO, I.B.M. Estatística aplicada à experimentação animal. Belo Horizonte: Fundação de Ensino e Pesquisa em Medicina Veterinária e Zootecnia, 2002. 265p.

SCHMIDTMANN, E.T.; MARTIN, P.A.W. Relationship between selected bacteria and the growth of immature house 
flies, Musca domestica, in an axenic test sistem. Journal of Medical Entomology, v.29, n.2, p.232-235, 1992. Disponível em: <http://esa.publisher.ingentaconnect.com/content/esa/jme/ 1992/00000029/00000002/art00016>. Acesso em: 04 jul. 2010 .

SMITH, J.; GRULA, E.A. Toxic components on the larval surface of the corn earworm (Heliothis zea) and their effects on germination and growth of Beauveria bassiana. Journal of Invertebrate Pathology, v.39, n.1, p.15-22, 1982. Disponível em: <http://www.sciencedirect.com/ science?_ob=PublicationURL\&_tockey $=\% 23$ TOC $\% 236888 \%$ $231982 \% 23999609998 \% 23542234 \% 23$ F L $\mathrm{P} \% 23 \&$ \& cdi=6888\&_pubType $=\mathrm{J} \& \mathrm{view}=\mathrm{c} \&$ \& auth=y\& _a c ct $=$ C $000050221 \&$ _version $=1 \&$ _url Version $=0$ \&_userid=10\&md5=847dd964312c5aa7baf43946b578be04>. Acesso em: 04 jul. 2010. doi: 10.1016/0022-2011(82)90153-7.

SMITS, N. et al. Comparison of non-linear temperature-dependent development rate models applied to in vitro growth of entomopathogenic fungi. Micological Research, v.107, p.14761484, 2003. Disponível em: <http://www.sciencedirect.com/ science?_ob=ArticleURL\&_udi=B7XMR-4RRXX79-

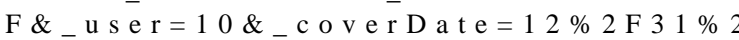
F2003\&_rdoc $=13 \&$ \&mt $=$ high\&_orig=browse\&_srch=docinfo ( \% 23 t o c \%232967 \% \% $232003 \% 2399892$ $9987 \% 23679648 \% 23$ F L P \% 23 display \%23 Volume )\&_cdi=29677\&_sort $=$ d\&_docanchor $=\&$ view $=$ c\&_ct $=1$ $5 \&$ \&_a c c t = C $000050221 \&$ \&_versi on $=1$ \&_url V ersion=0\&_userid=10\&md5=cb4f48f00bec2e4fa356bd2d8384cb43>. Acesso em: 04 jul. 2010. doi: 10.1017/S095375620300844X.

SOULSBY, E.J.L. Parasitología y enfermidades parasitarias en los animales domésticos. 7.ed. México: Nova Editorial Interamericana, 1987. 823p.

ST. LEGER, R.J. et al. Ambient pH is a major determinant in the expression of cuticle-degrading enzimes and hidrophobin by Metarhizium anisopliae. Applied Environmental
Microbiology, v.64, n.2, p.709-713, 1998. Disponível em: <ht t p://a em.asm.org/cgi/content/full/64/2/ 709 ? $\mathrm{maxt}$ os how $=\&$ hits $=10$ \& R E S U T F O R M $\mathrm{AT}=1 \&$ andorexacttitle $=$ and $\&$ andorexacttitleabs $=$ and $\&$ an dorexactfulltext $=$ and $\&$ searchid $=1 \&$ FIRSTINDE $X=0$ \&sortspec $=$ relevance $\&$ volume $=64 \&$ firstpage $=709 \&$ tdate $=7 /$ 31/1999\&resourcetype=HWCIT $>$. Acesso em: 04 jul. 2010. doi: 0099-2240/98/\$04.00+0

TARRY, D.W.; CARROLL, P.J. Summer mastitis: transmission by blood feeding flies. Veterinary Record, v.123, p.304, 1988 Disponível em: <http://veterinaryrecord.bvapublications.com/cgi/ gca ?allch $=$ \&SEARCHID $=1 \&$ VOL UME $=123 \&$ FIRS TPA GE $=304 \&$ F I R S T I N D E X $=0$ \& h it s $=10$ \& R E S ULTFORMAT=1\&gca=vetrecord\%3B $123 \% 2$ F11\%2F304b\#123/11/304-b>. Acesso em: 04 jul. 2010.

VILCINSKAS, A.; WEDDE, M. Inhibition of Beauveria bassiana proteases and fungal development by inducible protease inhibitors in the haemolymph of Galleria mellonella larvae. Bicontrol Science and Technology, v.7, p.501-601, 1997. Disponível em: <http://www.informaworld.com/smpp/ content $\sim \mathrm{db}=\mathrm{all} \sim \mathrm{content}=\mathrm{a} 713655851>$. Acesso em: 04 jul. 2010. doi: 10.1080/09583159730640.

WATSON, D.W. et al. Efficacy of Beauveria bassiana for controlling the house fly and stable fly (Diptera: Muscidae). Biological Control, v.5, p.405-411, 1995. Disponível em: <http://www.sciencedirect.com/ science?_ob=ArticleURL\&_udi=B6WBP-45N4NV4$1 \mathrm{~J} \&$ _user $=10$ \&_coverDate $=09 \% 2 \mathrm{~F} 30 \% 2 \mathrm{~F} 1995 \&$ \& rdoc $=1 \&$ _ $\mathrm{fmt}=$ high\&_orig=search\&_sort=d\&_docanchor=\&view $=$ c\&_searchS trId $=1391360406 \&$ rerunOrigin $=$ google\&_acct $=C 000050221$ \&_versi o $n=1 \&$ url Versio $n=0 \&$ us e ri d $=10 \& m$ d5=b8463486c2e11780aca2ccfeddd992b1>. Acesso em: 02 jul. 2010. doi: 10.1006/bcon.1995.1048.

WHITE, S.D.; BOURDEAU, P. Hypersensibilités aux piqûres de diptères chez les carnivores. Le Point Veterinaire, v.27, n.169, p.203-206, 1995. 